\title{
Establishment of a biomarker model for predicting bone metastasis in resected stage III non-small cell lung cancer
}

\author{
Zhen Zhou ${ }^{1,3^{*}}$, Zhi-Wei Chen ${ }^{1}$, Xiao-Hua Yang ${ }^{2}$, Lan Shen ${ }^{1}$, Xing-Hao Ai ${ }^{1}$, Shun Lu ${ }^{1}$ and Qing-Quan Luo
}

\begin{abstract}
Background: This study was designed to establish a biomarker risk model for predicting bone metastasis in stage III non-small cell lung cancer (NSCLC).

Methods: The model consists of 105 cases of stage III NSCLC, who were treated and followed up. The patients were divided into bone metastasis group $(n=45)$ and non-bone metastasis group (other visceral metastasis and those without recurrence) $(n=60)$. Tissue microarrays were constructed for immunohistochemical study of 10 molecular markers associated with bone metastasis, based on which a model was established via logistic regression analysis for predicting the risk of bone metastases. The model was prospectively validated in another 40 patients with stage III NSCLC.

Results: The molecular model for predicting bone metastasis was logit $(P)=-2.538+2.808$ CXCR4 +1.629 BSP +0.846 OPN-2.939 BMP4. ROC test showed that when $P \geq 0.408$, the sensitivity was up to $71 \%$ and specificity of $70 \%$. Model validation in the 40 cases in clinical trial (NCT 01124253) demonstrated that the prediction sensitivity of the model was $85.7 \%$, specificity $66.7 \%$, Kappa: 0.618 , with a high degree of consistency.
\end{abstract}

Conclusion: The molecular model combining CXCR4, BSP, OPN and BMP4 could help predict the risk of bone metastasis in stage IIla and IIIb resected NSCLC.

Keywords: Lung cancer, Bone metastasis, Biomarker

\section{Introduction}

Tumor cells homing to form bone metastases is common in non-small cell lung cancer (NSCLC), just like what is seen in breast, prostate and thyroid cancers. Some patients may experience bone metastasis many years after surgery of the primary tumor. The high morbidity and significantly increased risk of fractures associated with bone metastasis seriously affect patients' quality of life. About $36 \%$ of all lung cancers and and $54.5 \%$ of stage II-IIIA NSCLC showed postoperative recurrence or metastasis [1]. Many lung cancer patients expect new and more sensitive

\footnotetext{
* Correspondence: zzhou1116@hotmail.com

'Department of Lung Tumor Clinical Medical Center, Shanghai Chest Hospital affiliated to Shanghai Jiaotong University, and Thoracic Tumor Clinical Medicine Center of Shanghai Municipality, Shanghai, 200032, China

${ }^{3}$ Department of Lung Tumor Clinical Medical Center, Shanghai Chest Hospital affiliated to Shanghai Jiaotong University, and Thoracic Tumor Clinical Medicine Center of Shanghai Municipality, No 241, West Huaihai Road, Xuhui District, Shanghai 200032, China

Full list of author information is available at the end of the article
}

markers to predict metastatic diseases. If bone metastasis can be predicted early enough, then effective prevention could be started and may result in an improvement in survival [2]. The molecular and cellular mechanisms leading to the development of bone metastasis in NSCLC remain unclear, therefore in this study, we investigated the current understanding of bone metastasis in NSCLC. We constructed tissue microarray, and used immunohistochemical method to assess the expression of 10 bone metastasisrelated tumor markers in primary NSCLC tissue, which involved multi-step process of bone metastasis [3], including the proliferation, adhesion, escape (MMPs, OPN, c$\mathrm{Src})$ of primary tumors; targeted metastasis to bone (CXCR4); bone-specific adhesion and implantation (BSP); formation of metastases in bone (IGF1R, BMPs, PTHrP) and metastasis-associated cell signaling pathways (PI3K, $\mathrm{NF \kappa B}$ ). We established a molecular model composed of biological markers to predict the risk of bone metastasis in

\section{Biomed Central}


resected stage III NSCLC to screen the patients at high risk of bone metastasis for early intervention.

\section{Patients and methods Patients}

The patients for establishing the model were 105 cases of pathologically-confirmed stage III NSCLC, who were the whole cohort and treated by complete resection from June 2002 to December 2006 at Shanghai Chest Hospital, and were followed up until December 2008. Before surgery, these patients did not have any chemo/radiotherapy, immunotherapy or other treatments that could significantly modulate the cancer cell biology. All the patients had complete resection of the tumor and staged accoding UICC 1999. The patients included 65 males and 40 females. The median age was 59 (34 to 76) years. Pathological examination showed 88 cases of adenocarcinoma, and 17 cases of non-adenocarcinoma. Stage IIIa was confirmed in 86 cases, and IIIb in 19 cases. Cisplatin-based adjuvant chemotherapy was administrated to patient with completely resected NSCLC. Three or more cycles of postoperative adjuvant chemotherapy were received in 76 cases. The 45 cases of bone metastasis were designated as bone metastasis group. The remaining 60 cases with visceral metastasis or without metastasis were defined as non-bone metastasis group.

The patients recruited in the validation group in the prospective model consists of 40 cases of pathologically-confirmed Stage III NSCLC the whole cohort enrolled in clinical trial (NCT 01124253), who had received complete surgical resection from July 2007 to August 2009, 26 males and 14 females. The median age was 57 (41 to 76) years. Pathological examination showed 33 cases of adenocarcinoma, and 7 cases of non-adenocarcinoma. Stage IIIa was confirmed in 35 cases, and IIIb in 5 cases.

\section{Preparation of tissue microarray}

HE sections were examined under a microscope to identify and mark the cancer nests. HE sections were used to mark the corresponding sampling site on paraffin blocks of the donor. Preparation of tissue chip block: The ordinary pathological paraffin was melted and precipitated repeatedly for 3 times. Then $3 \%$ refined beeswax was added to prepare blank paraffin blocks of $32 \mathrm{~mm} \times 15$ $\mathrm{mm} \times 10 \mathrm{~mm}$, which were cooled at room temperature. Design and preparation of tissue microarray: an $8 \times 5$ tissue array was designed and made into module with the drilling system. A punch needle with a diameter of $2 \mathrm{~mm}$ was used to remove the tissue cores one by one from the specified site of donor paraffin blocks. The tissue cores were put into a pre-designed array module, arranged as tissue microarray. The prepared tissue microarrays were placed in an instrument at $60^{\circ} \mathrm{C}$ for $20 \mathrm{~min}$. The modules were pressed slightly to make the tissue cores level and align in the module. The prepared module was then cut into conventional $2 \mu \mathrm{m}$ slice and mounted on a silica slide. The slide was incubated overnight at $60^{\circ} \mathrm{C}$. Each chip of the tissue microarray contained the 20 tissue samples and 2 normal controls. Each case repeated.

\section{Immunohistochemistry S-P assay}

Mouse anti-human monoclonal antibodies against parathyroid hormone related protein (PTHrP), osteopontin (OPN), Src proto-oncogene (c-Src), matrix metalloproteinase - 2 (MMP2), chemokine receptor type 4 (CXCR4), phosphatidylinositol kinase (PI3K), bone sialoprotein (BSP), nuclear transcription factor (NFKB), insulin-like growth factor-IR (IGF-1R), bone morphogenetic protein -4 (BMP-4) were purchased from Beijing Boao Sen Biotechnology Co., Ltd. SP kit was purchased from Fuzhou Maixin Biotechnology Development Co., Ltd. Antigen retrieval was conducted according to the protocol. The known positive tissue sections of lung cancer were used as positive control, PBS instead of primary antibody as negative control. Evaluation criteria: immunostaining score was calculated based on the sum of the percent positivity of stained tumour cells and the staining intensity. The percent positivity was scored as "0" ( $<5 \%$, negative), "1" (5-25\%, sporadic), " 2 " (25-50\%, focal), "3" ( $>50 \%$, diffuse). The staining intensity was scored as "0" (no staining), "1" (weakly stained), "2" (moderately stained), and "3" (strongly stained). Both percent positivity of cells and staining intensity were decided under doubleblind condition. The final expression score was calculated with the value of percent positivity score $\times$ staining intensity score, which ranged from 0 to 9 . We defined expression level as follow: "” (score 0-1), "+" (score 2-3), "++" (score 4-6) and "+++" (score >6) [4]. Expression (++) or more be considered positive.

\section{Follow-up and database construction}

All patients were followed-up regularly by a designated staff, who collected all the information to a central database. Generally, we followed up the patients 3 to 4 times a year in the first 2 years, and once in half a year in the following 3 years. The disease control time (DCT) was defined as the time interval from surgical section to the recurrence. The last follow-up visit was on June 30, 2008. The DCT and site of recurrence were followed-up in the same way in validation group, for whom the date of last follow-up was June 30, 2011.

\section{Statistical analysis}

SPSS 17.0 software was used for data analysis. Categorical data were described by percentage to test group differences. Logistic regression analysis was applied to estimate the parameters using maximum likelihood estimation method, $\alpha=0.05$, for establishing a model to predict the risk of bone metastasis in resected stage III NSCLC. Model 
fitting was evaluated by Hosmer-Lemeshow test. The model was also tested by receiver operating characteristics (ROC) analysis, and prospectively validated with kappa test. $\mathrm{P}<0.05$ was considered statistically significant.

\section{Results}

\section{Model group}

A total of 105 cases of stage III NSCLC patients were analyzed, including 45 cases with bone metastasis, and 60 cases without bone metastasis. Only pathologic stage statistically significant difference was found between bone metastasis group and non-bone metastasis group in terms of clinical and pathological factors (Table 1).

\section{Establishment of the prediction model of bone metastasis} A number of cancer molecular markers associated with bone metastasis were assessed by immunohistochemical technique, including PTHrP, OPN, c-Src, MMP2, CXCR4, PI3K, BSP, NFkB, IGF-1R, and BMP4. Immunohistochemically, PTHrP, OPN, c-Src, MMP2, CXCR4, BSP, NFkB, IGF $-1 \mathrm{R}$, and BMP4 were mainly expressed in cytoplasm. PI3K was mainly expressed in cytoplasm, partly in the nucleus; BMP4expressed slight weakly (Figure 1). Chi-square $\left({ }^{2}\right)$ test showed that OPN, CXCR4, BSP, BMP4 were associated with bone metastasis (Table 2). A prediction model was established via Logistic regression analysis: logit $(P)=-$ $2.538+2.808$ CXCR4 +1.629 BSP +0.846 OPN-2.939 BMP4. Hosmer and Lemeshow test $p=0.065$. ROC test (Figure 2) suggested that the area under the curve was $81.5 \%$ ( $\mathrm{P}$ : 0.041 , 95\% CI $73.4 \%$ to $89.5 \%$ ). When $\mathrm{P}=0.408$, the sensitivity was up to $71 \%$, specificity $70 \%$. Namely, $\mathrm{P} \geq 0.408$ can be used as the screening indicator in this model to identify those at high risk of bone metastasis in resected stage III NSCLC.

\section{Prospective validation of bone metastasis prediction model}

A total of 40 cases of stage III NSCLC were enrolled from July 2007 to August 2009. TMA was constructed in Dec.2010 and assessed for OPN, CXCR4, BSP and BMP4. According to this model, we predicted 8 cases would have bone metastasis and 32 cases would not. Bone metastasis was identified in 7 (17.5\%) cases. Other visceral metastasis was found in 20 (50\%) cases. No metastasis was identified in $13(32.5 \%)$ cases. The prediction sensitivity of the model was $85.7 \%$, specificity of $66.7 \%$, Kappa: 0.618 , with a high degree of consistency.

\section{Discussion}

Bone metastases are classified as osteolylic, osteoselerotic or mixed lesions. Several molecular mechanism bring about cancer cell to metastasis to bone, and osteotropric cancer cells are believed to acquire bone cell-like properties which improve homing, adhesion, proliferation and survival in the bone microenvironment [2].

We used tissue microarray technology in this study. It is a good solution to a large volume of tumor marker tests and the comparability of results. Immunohistochemical assay was used to detect the expression of 10 molecular markers in 105 patients completely resected stage III with NSCLC tissue from the 2002 to 2006 the whole cohort. These molecular markers included PTHrP, OPN, c-Src, MMP2, CXCR4, PI3K, BSP, NFkB, IGF-1R, and BMP4. All these molecules may, individually, play important roles in breast cancer or prostate cancer bone metastasis. However, to our knowledge, there have been

Table 1 Comparison of major clinico-pathological factors between NSCLC patients with or without bone metastasis

\begin{tabular}{|c|c|c|c|}
\hline Characteristics & $\begin{array}{l}\text { Bone metastasis }(n=45) \\
n(\%)\end{array}$ & $\begin{array}{l}\text { Non-bone metastasis }(n=60) \\
n(\%)\end{array}$ & $\begin{array}{l}P \\
\text { value }\end{array}$ \\
\hline \multicolumn{4}{|l|}{ Gender } \\
\hline Male & $28(62.2)$ & $37(61.7)$ & \multirow[t]{2}{*}{0.954} \\
\hline Female & $17(37.8)$ & $23(38.3)$ & \\
\hline Age (mean $\pm S D)(y r)$. & $55.8 \pm 12.1$ & $57.4 \pm 7.2$ & 0.398 \\
\hline \multicolumn{4}{|l|}{ Histopathology } \\
\hline Adenocarcinoma & 39 (86.7) & $50(83.5)$ & \multirow[t]{2}{*}{0.567} \\
\hline Non-adenocarcinoma & $6(13.3)$ & $10(16.7)$ & \\
\hline \multicolumn{4}{|l|}{ Stage } \\
\hline Illa & $33(73.3)$ & $53(86.7)$ & \multirow[t]{2}{*}{0.048} \\
\hline$\| l l b$ & $12(26.7)$ & $7(13.3)$ & \\
\hline \multicolumn{4}{|l|}{ Adjuvant chemotherapy } \\
\hline Yes & $30(66.7)$ & $46(76.7)$ & \multirow[t]{2}{*}{0.828} \\
\hline No & $15(33.3)$ & $14(23.3)$ & \\
\hline
\end{tabular}




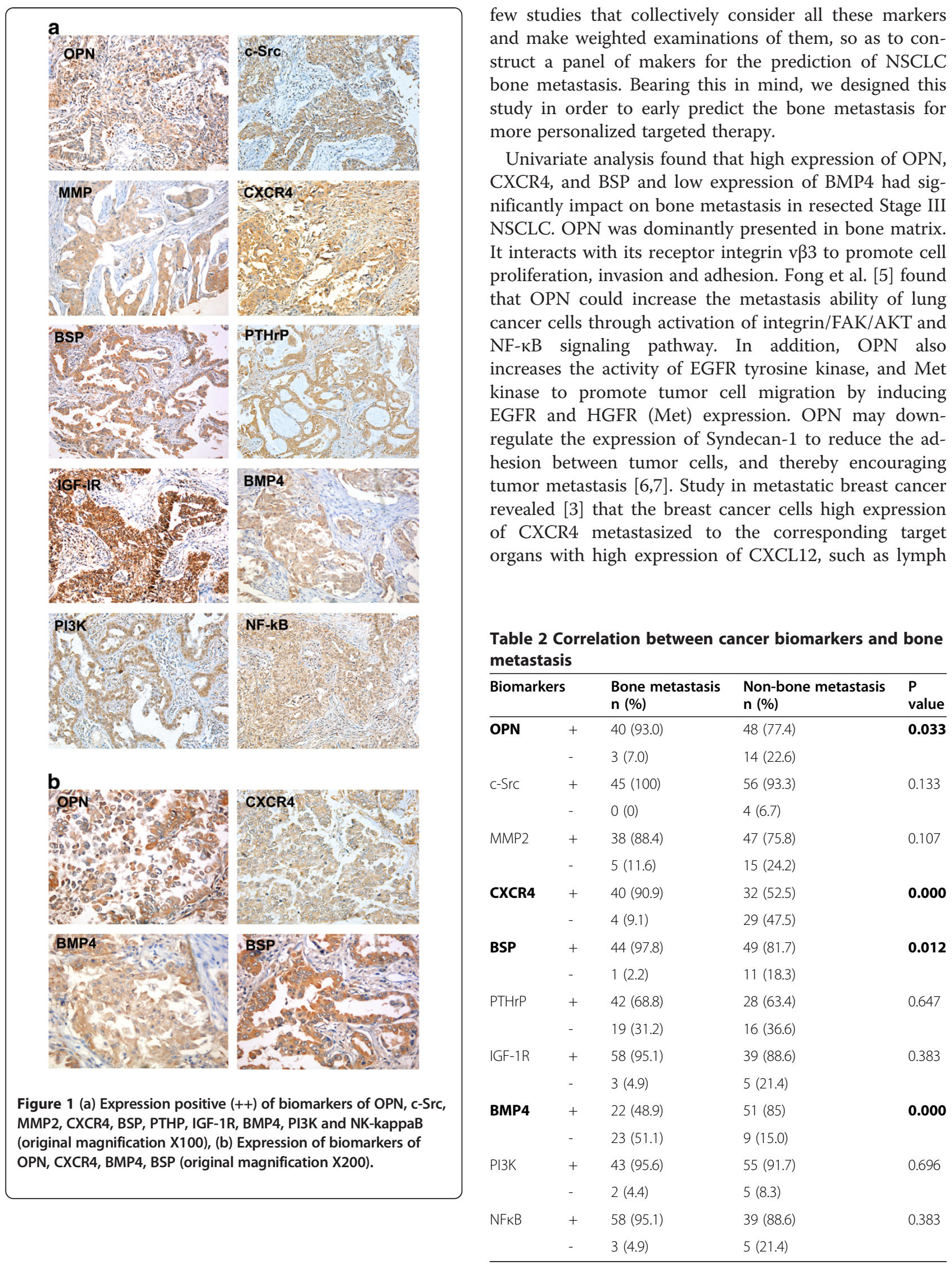




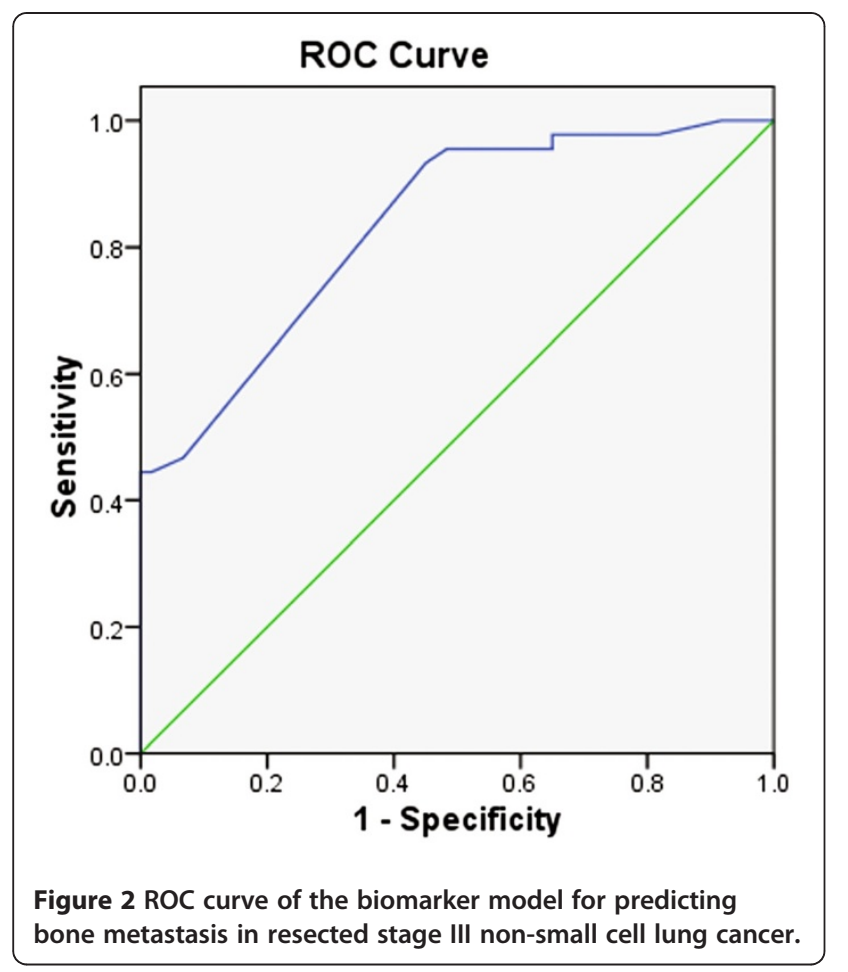

nodes and bone marrow. CXCR4 interacts with CXCL12 to promote tumor cell proliferation, induce the expression of MMP2, and increase invasion and metastasis. High expression of CXCR4 not only enhances distant metastasis, but also leads to more pronounced bone metastasis relative to visceral metastasis. In vitro experiments also confirmed that the high expression of CXCR4 alone was significantly associated with higher rate of bone metastasis [8]. No significant difference was found between bone metastasis group and non-bone metastasis group in this study, although MMP2 was over expressed in the patients with distant metastasis. According to the "seed and soil" theory, some tumor cells prefer to bone metastases due to their inherent biological characteristics, BSP and c-Src for example. BSP has cell adhesion function, and is involved in cell migration and signal recognition [9]. BSP acts as the ligand of integrin in osteoblasts, osteoclasts, and tumor cells of bone metastasis. It binds with integrin and play a role in osteocyte differentiation, bone matrix mineralization, as well as adhesion, proliferation and metastasis of tumor cells $[10,11]$. In the distant metastasis of both breast cancer and prostate cancer, the incidence of bone metastasis is higher than that of visceral metastasis in case of high expression of BSP. The level of BSP expression in the cancer cells of bone metastasis is higher than that in the primary tumor. The primary tumor with high expression of BSP may be more inclined to bone as a target organ of metastasis. And the microenvironment of bone further up-regulates the expression of the BSP, while the microenvironment of visceral organs reduces the expression of BSP. Positive expression of BSP in tumor cells suggests that BSP has contributed to bone metastasis [12]. Studies comparing bone and visceral metastasis in breast cancer indicated that up-regulation of c-Src gene can increase bone metastasis, while down-regulation of this gene will decrease the malignant phenotype of breast cancer cells, and reduce bone metastasis [13]. However, high expression of c-Src was not associated with stronger bone metastasis than other distant metastasis in this study. BMPs are bone morphogenetic proteins, which is a member of growth factor family. Functional studies have shown that BMPs are involved in both promotion and inhibition of tumor cell growth [14]. BMPs secreted by tumor cells can induce cell differentiation of osteoblasts, increase the formation of new bone and promote bone mineralization. Recent evidence suggests [15] that the BMP4-induced phenotypic changes were mediated through the activation of the canonical SMAD signaling pathway. It has been reported that BMP4 is overexpressed in melanoma cell line and lung cancer. BMP4 plays an important role in bone metastasis of prostate cancer [16], and BMP4 overexpression inhibits proliferation and induces apoptosis in many cancer cell line $[15,17]$. This study also showed that BMP-4 expression was lower in primary tumors. Bone metastasis of lung cancer is a dynamic process involving bone resorption resulted from tumor cell-induced osteolysis and bone formation due to osteoblasts. This study didn't show PTHrP and IGF-1R overexpression in NSCLC tissue related NSCLC bone metastasis. PTHrP is required for colony of bone metastasis of cancer cells. It is a cytokine produced by the metastatic cancer cells [18]. But Henderson [19] had demonstrated that bone metastases that do not express PTHrP in primary breast cancer begin to do so when they reach bone. The bone microenvironment seems to provide what is needed for the breast cancer cells to produce PTHrP, even if they could not produce it before they got there. This study demonstrated that PTHrP was expressed only in $66.67 \%$ of the primary tumors. Breast cancer overexpress IGF-1R through promoting proliferation and reducing apoptosis to increase bone metastasis [20], the effects of IGF-1R have been confirmed in bone metastasis of prostate cancer [21] but the role of IGF-1R overexpress in NSCLC bone metastasis is not clear, it still needs to be further investigated.

Multivariate Logistic regression has successfully established a model for predicting the risk of bone metastasis in resected Stage III NSCLC: logit $(\mathrm{P})=-2.538+2.808$ CXCR4 +1.629 BSP +0.846 OPG-2.939BMP4. The area under the ROC curve was $81.5 \%$. When $\mathrm{P}=0.408$, the sensitivity was up to $71 \%$, specificity $70 \%$. The model has successfully validated in 40 patients with resected stage 
III NSCLC from 2007 to 2009 whole cohort in clinic trial, who were followed up for 3 years. The model showed a sensitivity of $85.7 \%$ and specificity of $66.7 \%$, Kappa: 0.618. The results are highly consistent. The model based on bone metastasis-associated biomarkers established in this study is useful in providing rationale for the screening, intervention and targeted therapy of bone metastasis in lung cancer.

Although the results are interesting, the limitations of this study should be acknowledged. The patients enrolled into the prediction model and validation model were whole cohort of completed resected stage III patients, not including patients from other groups. Therefore, there might be selection bias in the model construction and results interpretation. The results might be more suitable to clinically stage III patients. Any generalization to other stages should not be expected. In the future, a bigger study with larger sample size with different stages, could help more objectively judge the value of this prediction model.

\section{Competing Interests}

The authors have declared that no competing interests exist.

\section{Acknowledgments}

The study was support by the Shanghai Municipal Health Bureau Grand (2008146). We appreciate Dr. Meiling Liao and Dr. Jie Zhang for their help, as well as all patients and their families and the staffs participating in this research.

\section{Author details}

'Department of Lung Tumor Clinical Medical Center, Shanghai Chest Hospital affiliated to Shanghai Jiaotong University, and Thoracic Tumor Clinical Medicine Center of Shanghai Municipality, Shanghai 200032, China. ${ }^{2}$ Tumor Research Lab, Shanghai Chest Hospital affiliated to Shanghai Jiaotong University, Shanghai 200030, China. ${ }^{3}$ Department of Lung Tumor Clinical Medical Center, Shanghai Chest Hospital affiliated to Shanghai Jiaotong University, and Thoracic Tumor Clinical Medicine Center of Shanghai Municipality, No 241, West Huaihai Road, Xuhui District, Shanghai 200032, China.

\section{Authors' contributions}

ZZ carried out the protocol design, participated in the patients enrollment and TMA assay, drafted the manuscript. Z-WC carried out the patients enrollment. X-HY carried out the TMA immunohistochemistry assay. These authors contributed equally to this work. All authors read and approved the final manuscript.

Received: 31 January 2012 Accepted: 26 April 2012 Published: 26 April 2012

\section{References}

1. Coleman RE: Clinical features of metastatic bone disease and risk of skeletal morbidity. Clin Cancer Res 2006, 12:6243s-6249s.

2. Clezardin P, Teti A, et al: Bone metastasis: pathogenesis and therapeutic implications. Clin Exp Metastasis 2007, 24:599-608.

3. Zhang Y, Ma B, Fan Q: Mechanisms of breast cancer bone metastasis. Cancer Let 2010, 292:1-7.

4. Wang H, Pan K, Zhang HK, et al: Increased polycomb-group oncogene Bmi-1 expression correlates with poor prognosis in hepatocellular carcinoma. J Cancer Res Clin Oncol 2008, 134:535-541.

5. Fong YC, Liu SC, Huang CY, et al: Osteopontin increases lung cancer cells migration via activation of the $\mathrm{v} \beta 3$ integrin/FAK/Akt and NF-KB-dependent pathway. Lung Cancer 2009, 64:263-270.
6. El-Tanani MK: Role of osteopontin in cellular signaling and metastatic phenotype. Front Biosci 2008, 13:4276-84.

7. Wai PY, Guo L, Gao C, et al: Osteopontin inhibits macrophage nitric oxide synthesis to enhance tumor proliferation. Surgery 2006, 140:132-140.

8. Kang Y, Siegel PM, Shu W, et al: A multigenic program mediating breast cancer metastasis to bone. Cancer Cell 2003, 3:537-549.

9. Uemura T, Liu YK, Feng Y, et al: The role of sialoprotein in recognition of bone surface by osteoblasts via integrin. Mat Sci Eng 1997, 4:303.

10. Bellancene A, MerVille MP, Castronovo V: Expression of bone sialoprotein, a bone matrix protein, in human breast cancer. Cancer Res 1994, 54:2823.

11. Fp R, Chappel J, Alvarez Jl, et al: Interactions between the bone metrix proteins osteopontin and bone sialoprotein and the osteoclast integrin alpha v beta 3 potentiate bone resorption. J Biol Chem 1993, 268:9901-9907.

12. Waltregny $D$, Bellahcene $A$, de Leval $X$, et al: Increased Expression of bone sialoprotein in bone metastases compared with visceral metastases in human breast and prostate cancers. J Bone Miner Res 2000, 15:834-843.

13. Myoui A, Nishimura R, Williams PJ, et al: c-SRC tyrosine kinase activity is associated with tumor colonization in bone and lung in an animal model of human breast cancer metastasis. Cancer Res 2003, 63:5028-5033.

14. Alarmo EL, Kallioniemi A: Bone morphogenetic proteins in breast cancer: dual role in tumourigenesis? Endocr Relat Cancer 2010, 17:R123-R139.

15. Ketolainen JM, Alarmo EL, Tuominen VJ, et al: Parallel inhibition of cell growth and induction of cell migration and invasion in breast cancer cells by bone morphogenetic protein 4. Breast Cancer Res Treat 2010, 124:377-386.

16. Spanjol J, Djordjeric G, Markic D, et al: Role of bone morphogenetic proteins in human prostate cancer pathogenesis and development of bone metastases: immuohistochemical study. Coll Antropol 2010, 34(Suppl 2):119-125.

17. Hjertner $\mathrm{O}, \mathrm{Hj}$ th-Hansen $\mathrm{H}$, Borset $\mathrm{M}$, et al: Bone morphogenetic protein-4 inhibits proliferation and induces apoptosis of multiple myeloma cells. Blood 2001, 7:516-522.

18. Luparello C: Midregion PTHrP and human breast cancer cells. Sci World J 2010, 1:1016-1028.

19. Henderson MA, Danks JA, Slavin JL, et al: Parathyroid hormone related protein localization in breast cancers predict improved prognosis. Cancer Res 2006, 66:2250-2256.

20. Yoneda T, Hiraga T: Crosstalk between cancer cell and bone microenviroment in bone metastasis. Biochem Biophys Res Commun 2005, 328:679-687.

21. Yonou $H$, Ogawa $Y$, Ochiai A: Mechanism of osteoblastic bone metastasis of prostate cancer. Clin Calcium 2006, 16:557-564.

doi:10.1186/1756-9966-31-34

Cite this article as: Zhou et al.: Establishment of a biomarker model for predicting bone metastasis in resected stage III non-small cell lung cancer. Journal of Experimental \& Clinical Cancer Research 2012 31:34.

\section{Submit your next manuscript to BioMed Central and take full advantage of:}

- Convenient online submission

- Thorough peer review

- No space constraints or color figure charges

- Immediate publication on acceptance

- Inclusion in PubMed, CAS, Scopus and Google Scholar

- Research which is freely available for redistribution 